PAPER

\section{Analysis of ELM stability with extended MHD models in JET, JT-60U and future JT-60SA tokamak plasmas}

To cite this article: N Aiba et al 2018 Plasma Phys. Control. Fusion 60014032

View the article online for updates and enhancements.

\section{Related content}

- Impact of ion diamagnetic drift on MHD
$\frac{\text { stability at edge pedestal in JT-60U }}{\text { rotating plasmas }}$
N. Aiba, M. Honda and K. Kamiya
$-\frac{\text { Numerical analysis of ELM stability with }}{\text { rotation and ion diamagnetic drift effects in }}$
$\frac{\text { JET }}{\text { N. Aiba, C. Giroud, M. Honda et al. }}$
$-\frac{\text { Impact of ion diamagnetic drift on ideal }}{\text { ballooning mode stability in rotating }}$
$\frac{\text { tokamak plasmas }}{\text { N Aiba }}$

\section{Recent citations}

- Advances in the physics studies for the JT-
$\frac{\text { 60SA tokamak exploitation and research }}{\text { plan }}$
G Giruzzi et al
- Non-linear magnetohydrodynamic
$\frac{\text { simulations of pellet triqgered edge- }}{\text { localized modes in JET }}$
S. Futatani et al
- Direct gyrokinetic comparison of pedestal
$\frac{\text { transport in JET with carbon and ITER-like }}{\text { walls }}$
D.R. Hatch et al




\title{
Analysis of ELM stability with extended MHD models in JET, JT-60U and future JT-60SA tokamak plasmas
}

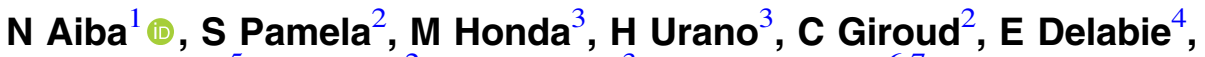 \\ L Frassinetti $^{5}$, I Lupelli ${ }^{2}$, N Hayashi ${ }^{3}$, G Huijsmans $^{6,7}$, the JET Contributors ${ }^{8}$ \\ and JT-60SA Research Unit ${ }^{9}$

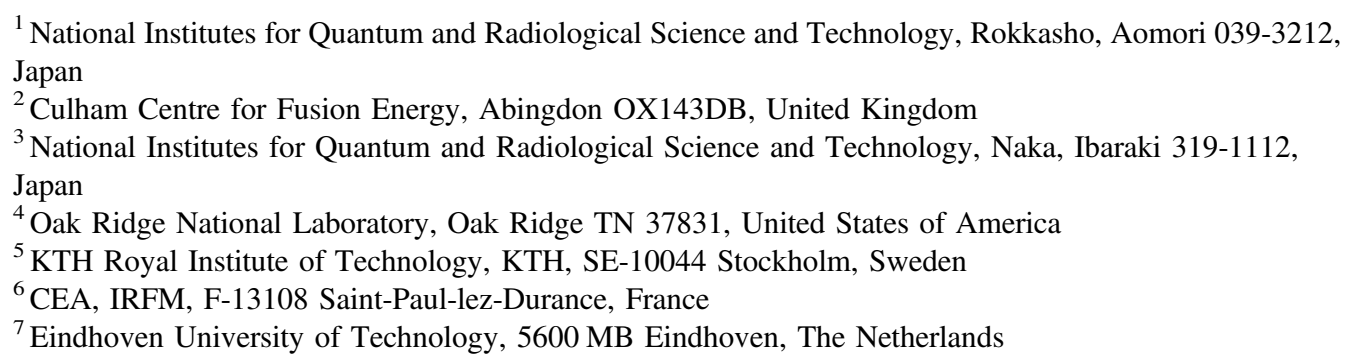 \\ E-mail: aiba.nobuyuki@qst.go.jp
}

Received 23 June 2017, revised 8 September 2017

Accepted for publication 12 September 2017

Published 7 November 2017

\begin{abstract}
The stability with respect to a peeling-ballooning mode (PBM) was investigated numerically with extended MHD simulation codes in JET, JT-60U and future JT-60SA plasmas. The MINERVA-DI code was used to analyze the linear stability, including the effects of rotation and ion diamagnetic drift $\left(\omega_{* \mathrm{i}}\right)$, in JET-ILW and JT-60SA plasmas, and the JOREK code was used to simulate nonlinear dynamics with rotation, viscosity and resistivity in JT-60U plasmas. It was validated quantitatively that the ELM trigger condition in JET-ILW plasmas can be reasonably explained by taking into account both the rotation and $\omega_{* i}$ effects in the numerical analysis. When deuterium poloidal rotation is evaluated based on neoclassical theory, an increase in the effective charge of plasma destabilizes the PBM because of an acceleration of rotation and a decrease in $\omega_{* \mathrm{i}}$. The difference in the amount of ELM energy loss in JT-60U plasmas rotating in opposite directions was reproduced qualitatively with JOREK. By comparing the ELM affected areas with linear eigenfunctions, it was confirmed that the difference in the linear stability property, due not to the rotation direction but to the plasma density profile, is thought to be responsible for changing the ELM energy loss just after the ELM crash. A predictive study to determine the pedestal profiles in JT-60SA was performed by updating the EPED1 model to include the rotation and $\omega_{* i}$ effects in the PBM stability analysis. It was shown that the plasma rotation predicted with the neoclassical toroidal viscosity degrades the pedestal performance by about $10 \%$ by destabilizing the PBM, but the pressure pedestal height will be high enough to achieve the target parameters required for the ITER-like shape inductive scenario in JT-60SA.
\end{abstract}

Keywords: ELM, extended MHD model, rotation, tokamaks, H-mode

(Some figures may appear in colour only in the online journal)

\footnotetext{
8 See the author list of 'Overview of the JET results in support to ITER' by Litaudon et al 2017 Nucl. Fusion 57102001.

9 See the author list of JT-60SA Research plan v3.3 at http://jt60sa.org/ pdfs/JT-60SA_Res_Plan.pdf.
}

\section{Introduction}

The high-confinement-mode (H-mode) in tokamak plasmas is usually accompanied by edge localized modes (ELMs), and 
one of them, called a type-I ELM, creates periodic large heat loads on divertors. In future tokamak fusion reactors like ITER and DEMO, the amount of the heat loads is thought to be unacceptable because of the critical damage to divertor plates. Therefore, it is necessary to develop operation scenarios and/or control techniques in order to avoid such large ELM heat loads. The important presupposition required to achieve this objective is the precise prediction of the plasma conditions triggering the ELM, and many past works have shown that a stability analysis with the ideal MHD model has successfully explained the plasma conditions observed in the experiments [1-3]. These results proved that the type-I ELM is triggered by an ideal MHD mode, called a peelingballooning mode (PBM), and the trigger condition is determined by the amount of plasma pressure and current density near the edge transport barrier region (pedestal).

However, the type-I ELM could sometimes be observed experimentally in JT-60U and JET with an ITER-like wall (JET-ILW), even when the pedestal pressure is much lower than that predicted numerically $[3,4]$. The results imply that additional physics effects neglected in the ideal MHD model may be responsible for the ELM stability in these experiments. The main candidates of these effects are the ion diamagnetic drift $\left(\omega_{* \mathrm{i}}\right)$ effect [5], plasma rotation in the toroidal and poloidal directions [3,5], plasma resistivity [6], and viscosity/diffusivity [7]. Many works have qualitatively identified their impact on both the linear stability and nonlinear dynamics of ELMs with linear and nonlinear MHD simulation codes, such as ELITE [5], MINERVA [8], MINERVA-DI [9], JOREK [10], BOUT ++ [7, 11], NIMROD [12, 13], M3D [14, 15], M3D-C1 [6], and so on.

Some quantitative analyses, including part of these effects, have been performed in parallel by investigating the stability with respect to PBM in the equilibria with the plasma profiles measured experimentally. For example, the impact of plasma rotation on the ELM stability in JT-60U was identified with MINERVA [3], and the ELM stability in JT-60U and JET plasmas was validated again by including not only the rotation but also the $\omega_{* i}$ effects with the linear extended MHD stability code MINERVA-DI [16, 17]. Furthermore, the impact of the $\omega_{* \mathrm{i}}$, resistivity and viscosity on the ELM stability in JET-ILW has been investigated with the nonlinear extended MHD stability code JOREK [18]. The results showed that these physics effects contribute to determining the ELM trigger conditions in such large tokamak experiments.

In this paper, based on these understandings, we consider the impact of plasma rotation on the stability of PBM when additional physics effects are taken into account simultaneously. The numerical analyses were performed with the extended MHD codes MINERVA-DI and JOREK. In section 2, the basic equations used in MINERVA-DI and JOREK are briefly introduced. Section 3 shows the results of the quantitative analysis of the linear PBM stability in JET-ILW, including the plasma rotation and $\omega_{* \mathrm{i}}$ effects with MINERVA-DI. After that, we examine with JOREK the difference in the amount of ELM energy loss observed in JT-60U plasmas rotating in opposite directions in section 4. In this analysis, finite resistivity is included to simulate magnetic reconnection, which is necessary to realize convective heat transport from pedestal to scrape-off layer (SOL) regions. Based on the understandings obtained with the validation studies in the present experiments, we perform the first predictive study of the pedestal profiles in JT-60SA [19] by including the rotation and $\omega_{* \mathrm{i}}$ effects in the PBM stability analysis, and the result is introduced in section 5. Section 6 presents a summary and discussion of this study.

\section{Basic equations}

In this section, the basic equations of the linear and nonlinear extended MHD stability codes, MINERVA-DI and JOREK, are introduced briefly; the details are written in [9] and [18], respectively.

The MINERVA-DI code solves the extended FriemanRotenberg equation, which is the linearized equation of motion of the diamagnetic MHD model

$$
\begin{aligned}
& \rho_{0} \frac{\partial^{2} \boldsymbol{\xi}}{\partial t^{2}}+2 \rho_{0}\left(\boldsymbol{V}_{0, \mathrm{MHD}} \cdot \nabla\right) \frac{\partial \boldsymbol{\xi}}{\partial t}+\rho_{0}\left(\boldsymbol{V}_{0, * \mathrm{i}} \cdot \nabla\right) \frac{\partial \boldsymbol{\xi}_{\perp}}{\partial t} \\
& =\boldsymbol{F}_{\mathrm{MHD}}+\boldsymbol{F}_{* \mathrm{i}}, \quad \\
& \nabla \cdot \boldsymbol{\xi}=0,
\end{aligned}
$$

with the definition of velocity vectors as

$$
\begin{gathered}
\boldsymbol{V}_{\mathrm{MHD}}=\boldsymbol{V}_{E}+V_{\|} \frac{\boldsymbol{B}}{|\boldsymbol{B}|}, \\
\boldsymbol{V}_{E}=\frac{\boldsymbol{E} \times \boldsymbol{B}}{B^{2}}, \\
\boldsymbol{V}_{* \mathrm{i}}=\frac{1}{e \bar{Z} N B^{2}} \boldsymbol{B} \times \nabla p_{\mathrm{i}},
\end{gathered}
$$

where $\boldsymbol{F}_{\mathrm{MHD}}$ and $\boldsymbol{F}_{* \mathrm{i}}$ are the force operators coming from the ideal MHD and ion diamagnetic correction parts; the third term on the left hand side of (1) and $\boldsymbol{F}_{* \mathrm{i}}$ appears by including the lowest order ion diamagnetic correction terms into the ideal MHD model [9]. Here $\rho$ is the mass density, $\boldsymbol{\xi}$ is the Lagrangian displacement vector defined with the linearized Eulerian velocity as

$$
\boldsymbol{V}_{1, \mathrm{MHD}} \equiv \frac{\partial \boldsymbol{\xi}}{\partial t}+\left(\boldsymbol{V}_{0, \mathrm{MHD}} \cdot \nabla\right) \boldsymbol{\xi}-(\boldsymbol{\xi} \cdot \nabla) \boldsymbol{V}_{0, \mathrm{MHD}},
$$

the subscript 0 (1) indicates the equilibrium (perturbed) quantity, $\boldsymbol{B}$ is the magnetic field, $\boldsymbol{E}$ is the electric field, $e$ is the quantum of electricity, $\bar{Z}$ is the ion mean charge satisfying $N_{\mathrm{e}}=\bar{Z} N, N_{\mathrm{e}}(N)$ is the electron (ion) number density, and $p_{\mathrm{i}}$ is the ion pressure. It should be noted that the incompressible assumption (2) and the flute approximation $\left(\boldsymbol{B}_{0} \cdot \nabla\right) \boldsymbol{\xi} \ll 1$, which are sometimes imposed in the extended MHD models (e.g. [20]), are introduced for deriving (1). The original diamagnetic MHD model was developed to investigate the impact of the $\omega_{* i}$ effect on ideal MHD stability in rotating plasmas; hence, MINERVA-DI allows us to analyze the linear stability of PBM with the rotation and 
$\omega_{* \mathrm{i}}$ effects, where $\omega_{* \mathrm{i}} \equiv \boldsymbol{V}_{0, * \mathrm{i}} \cdot \boldsymbol{k}$, and $\boldsymbol{k}$ is the wave number vector.

The basic equations of the JOREK code are the following five-field reduced MHD equations

$$
\begin{gathered}
\rho \frac{\partial \boldsymbol{V}_{E}}{\partial t}+\rho\left(\left(\boldsymbol{V}_{E}+\boldsymbol{V}_{* \mathrm{i}}\right) \cdot \nabla\right) \boldsymbol{V}_{E} \\
=\boldsymbol{j} \times \boldsymbol{B}-\nabla_{\perp} p+\mu \nabla^{2}\left(\boldsymbol{V}_{E}+\boldsymbol{V}_{* \mathrm{i}}\right), \\
\rho \frac{\partial \boldsymbol{V}_{\|}}{\partial t}+\rho\left(\boldsymbol{V}_{\|} \cdot \nabla\right) \boldsymbol{V}_{\|}=-\nabla_{\|} p+\mu \nabla^{2}\left(\boldsymbol{V}_{\|}-\boldsymbol{V}_{\mathrm{NBI}}\right), \\
\frac{\partial \psi}{\partial t}=\eta\left(j_{\phi}-j_{A}\right)+R[\psi, \Phi]-\frac{\partial \Phi}{\partial \phi} \\
-\frac{\delta^{*} R}{\rho}\left[\psi, p_{\mathrm{e}}\right]+\frac{\delta^{*}}{\rho} \frac{\partial p_{\mathrm{e}}}{\partial \phi} \\
\frac{\partial \rho}{\partial t}=-\nabla \cdot\left(\rho\left(\boldsymbol{V}_{E}+\boldsymbol{V}_{* i}\right)\right) \\
+\nabla \cdot\left(D_{\perp} \nabla_{\perp} \rho\right)+S_{\rho} \\
\frac{\partial p}{\partial t}=-\boldsymbol{V}_{E} \cdot \nabla p-\Gamma p \nabla \cdot \boldsymbol{V}_{E} \\
+\nabla \cdot\left(\kappa_{\perp} \nabla_{\perp} T+\kappa_{\|} \nabla_{\|} T\right)+S_{T}
\end{gathered}
$$

with the definition of the operators

$$
\begin{gathered}
\nabla_{\|} \equiv \frac{\boldsymbol{B}}{|B|}\left(\frac{\boldsymbol{B}}{|B|} \cdot \nabla\right), \\
\nabla_{\perp} \equiv \nabla-\nabla_{\|}, \\
{[\zeta, \lambda]=R^{2} \nabla \phi \cdot(\nabla \zeta \times \nabla \lambda) .}
\end{gathered}
$$

Here $\boldsymbol{j}$ is the current density, $p$ is the total pressure, $\mu$ is the viscosity, $\psi$ is the poloidal magnetic flux, $\eta$ is the resistivity, $j_{\phi}$ is the toroidal current density, $R$ is the major radius, $\Phi$ is the electric potential, $\phi$ is the toroidal angle, $\delta^{*} \equiv\left(\Omega_{c i} R_{o}\right), \Omega_{c i}$ is the ion gyrofrequency, $R_{o}$ is the reference major radius, $p_{\mathrm{e}}$ is the electron pressure, and $\Gamma=5 / 3$ is the specific heat ratio. The neutral beam injection (NBI) momentum, mass density, temperature, and current sources $\boldsymbol{V}_{\mathrm{NBI}}, S_{\rho}, S_{T}$ and $j_{A}$ have been introduced, where $j_{A}$ also includes the time-dependent bootstrap current calculated using Sauter's formula [21, 22]. The perpendicular mass and thermal diffusivities $D_{\perp}$ and $\kappa_{\perp}$ used in the simulations are ad hoc coefficients with a well at the pedestal region to represent the transport barrier, and the parallel thermal conductivity $\kappa_{\|}$is expressed to follow the Braginskii one as $\kappa_{\|}=\kappa_{\|, o}\left(T / T_{o}\right)^{5 / 2}$. The basic equations of JOREK include the effects of rotation, $\omega_{* i}$, resistivity, viscosity, and diffusivities, but in this study, we consider the impact of rotation on the nonlinear ELM dynamics with ad hoc fixed parameters $\eta=1.0 \times$ $10^{-6} \Omega \cdot \mathrm{m}, \mu=1.0 \times 10^{-7} \mathrm{~kg}(\mathrm{~m} \cdot \mathrm{s})^{-1}$, respectively. The $D_{\perp}$ and $\kappa_{\perp}$ values are determined to make $D_{\perp} \nabla \rho$ and $\kappa_{\perp} \nabla T$ constant in the pedestal region [18], and $D_{\perp}$ and $\kappa_{\perp}$ at $\psi=0.8$ are assumed to be $5.0 \mathrm{~m}^{2} \mathrm{~s}^{-1}$ and $1.0 \times 10^{-7}(\mathrm{~m} \cdot \mathrm{s})^{-1}$.

\section{ELM stability analysis with rotation and ion diamagnetic drift in JET-ILW}

In this section, we investigate the impact of plasma rotation and $\omega_{* \mathrm{i}}$ on the stability of MHD modes at the edge pedestal in JET-ILW. After installing ILW to JET, additional physics effects may be required to explain the ELM trigger condition when the fueling gas rate, $\Gamma_{D}$, is moderate to high with a high heating power, $P_{\mathrm{h}}$, although the ideal MHD stability can explain the condition when $\Gamma_{D}$ is low or $P_{\mathrm{h}}$ is low [4]. It was recently found that the PBM stability in JET with a carbon wall (JET-C) is hardly affected by rotation, but the rotation can destabilize the PBM and helps to explain the ELM trigger condition in JET-ILW with moderate to high- $\Gamma_{D}$ and high- $P_{\mathrm{h}}$ [17]. Such a difference is due to the fact that the rotation shear in JET-ILW plasmas was larger than that in JET-C ones, and the shear enhances the dynamic pressure destabilizing intermediate- $n$ MHD modes, where $n$ is the toroidal mode number. In section 3.1, we show the result of the validation study regarding the effects of plasma rotation and $\omega_{* \mathrm{i}}$ on the PBM stability in JET-ILW. After the validation, we confirm the sensitivity of the stability to the effective charge, $Z_{\text {eff }}$, when including these effects.

\subsection{Validation study regarding the impact of plasma rotation and ion diamagnetic drift on the ELM stability in JET-ILW}

The validation study has been performed by identifying the stability of PBM in 14 JET-ILW shots with different physics models; a summary of the shots is shown in table 1 . Here $B_{t 0}$ is the magnetic field on axis, $I_{\mathrm{p}}$ is the plasma current, $R_{0}$ is the major radius on axis, $\kappa$ is the ellipticity, $\delta$ is the triangularity, $\beta_{\mathrm{N}}$ is the normalized beta, $\nu_{* \mathrm{e}, 95}$ is the collisionality at $\psi=0.95$, respectively. These shots can be classified into two groups. One is a low- $\nu_{* e, 95}(<0.5)$ group obtained by moderate- $\Gamma_{D} \sim 12.0 \times$ $10^{21} \mathrm{el} \cdot \mathrm{s}^{-1}$ and high- $P_{\mathrm{h}} \sim 16.0 \mathrm{MW}$ with $B_{t 0}=1.9 \mathrm{~T}, I_{\mathrm{p}}=$ $1.4 \mathrm{MA}$ and $\delta \sim 0.26$, and the other is a high- $\nu_{* e, 95}(>0.8)$ one obtained by high- $\Gamma_{D}>20.0 \times 10^{21} \mathrm{el} \cdot \mathrm{s}^{-1}$ and high $-P_{\mathrm{h}}>$ $15.0 \mathrm{MW}$ with $B_{t 0}=2.6 \mathrm{~T}, I_{\mathrm{p}}=2.5 \mathrm{MA}$ and $\delta \sim 0.37$; only the \#89145 plasma, which has the lowest $\nu_{* \mathrm{e} .95}$ in the analyzed plasmas, was obtained with relatively low- $\Gamma_{D} \sim 6.0 \times$ $10^{21} \mathrm{el} \cdot \mathrm{s}^{-1}$. The models used to analyze the PBM stability are the ideal MHD model without rotation (IDEAL), and the diamagnetic MHD model with (DIAwR) and without rotation (DIAwoR), respectively. The range of the $n$ numbers of PBM analyzed numerically is between 1 and 100 , and the boundary condition is an ideal conducting wall located at $d / a=1.3$, where $a(d)$ is the plasma (wall) minor radius.

The ELM stability diagram is obtained by analyzing the stability in the equilibria whose pedestal pressure gradient and edge current density are changed by adjusting the height of the pressure pedestal and the amount of the bootstrap current near the pedestal; the details can be found in [16]. The bulk deuterium rotation profiles in both the toroidal and poloidal directions are evaluated with the CHARROT code from the measured profiles of plasma density, temperature, and impurity toroidal rotation, $\Omega_{\phi}$, by assuming that the neoclassical theory is applicable [16]. In this study, $\Omega_{\phi}$ of 
Table 1. Summary of the equilibrium parameters of the JET-ILW shots analyzed for the quantitative validation study regarding the impact of plasma rotation and $\omega_{* i}$ on ELM stability.

\begin{tabular}{lccccccccccc}
\hline Number & $B_{t 0}[\mathrm{~T}]$ & $I_{\mathrm{p}}[\mathrm{MA}]$ & $R_{0}[\mathrm{~m}]$ & $\kappa$ & $\delta$ & $\beta_{\mathrm{N}}$ & $\nu_{* \mathrm{e}, 95}$ & $Z_{\mathrm{eff}}$ & $\Gamma_{D}\left[\times 10^{21} \mathrm{el} \mathrm{s}^{-1}\right]$ & $P_{\mathrm{h}}[\mathrm{MW}]$ & Seeded impurity \\
\hline 89145 & 1.71 & 1.38 & 3.07 & 1.65 & 0.260 & 2.29 & 0.273 & 1.65 & 6.0 & 13.7 & none \\
90287 & 1.87 & 1.37 & 3.13 & 1.63 & 0.263 & 2.26 & 0.360 & 1.86 & 12.0 & 16.5 & none \\
90339 & 1.87 & 1.37 & 2.08 & 1.63 & 0.262 & 2.12 & 0.405 & 2.45 & 11.4 & 16.0 & Ne \\
90337 & 1.87 & 1.37 & 3.10 & 1.63 & 0.262 & 2.11 & 0.408 & 2.16 & 11.7 & 16.2 & $\mathrm{Ne}$ \\
90280 & 1.87 & 1.37 & 3.07 & 1.61 & 0.262 & 2.13 & 0.458 & 3.40 & 11.7 & 16.2 & Ne \\
82550 & 2.62 & 2.48 & 3.01 & 1.70 & 0.370 & 1.47 & 0.883 & 1.26 & 21.0 & 16.0 & none \\
87522 & 2.62 & 2.46 & 2.99 & 1.71 & 0.373 & 1.37 & 1.05 & 1.49 & 30.0 & 29.0 & Ne \\
82554 & 2.62 & 2.48 & 2.98 & 1.72 & 0.365 & 1.34 & 1.11 & 1.33 & 29.0 & 15.4 & $\mathrm{~N}$ \\
82551 & 2.62 & 2.49 & 2.99 & 1.73 & 0.365 & 1.48 & 1.13 & 1.35 & 21.7 & 15.6 & $\mathrm{~N}$ \\
87520 & 2.62 & 2.49 & 3.02 & 1.74 & 0.366 & 1.36 & 1.42 & 1.37 & 33.0 & 28.0 & Ne \\
89711 & 2.62 & 2.47 & 3.01 & 1.70 & 0.366 & 1.32 & 1.47 & 1.63 & 40.0 & 19.0 & $\mathrm{O}$ \\
89710 & 2.62 & 2.47 & 3.00 & 1.70 & 0.366 & 1.30 & 1.53 & 1.63 & 40.0 & 19.0 & $\mathrm{O}$ \\
89453 & 2.62 & 2.48 & 2.98 & 1.75 & 0.366 & 1.53 & 1.57 & 1.33 & 17.0 & 18.0 & $\mathrm{CD} 4$ \\
89709 & 2.62 & 2.47 & 3.00 & 1.70 & 0.366 & 1.28 & 1.73 & 1.50 & 40.0 & 19.0 & none \\
\hline
\end{tabular}

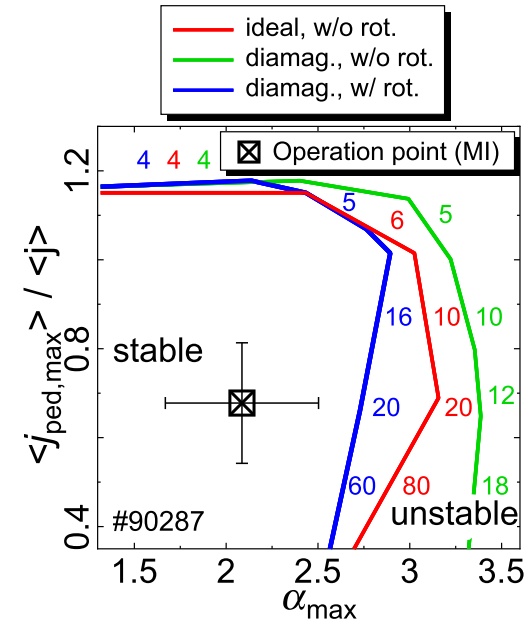

Figure 1. Stability diagram of the JET-ILW \#90287 low- $\nu_{* \mathrm{e}, 95}$ plasma on the $\left(\left\langle j_{\text {ped,max }}\right\rangle /\langle j\rangle, \alpha_{\text {max }}\right)$ plane. The stability boundaries are determined with the different models (IDEAL, DIAwoR and DIAwR). The width of the error bars on the operation point (O.P.) is determined to be $\pm 20 \%$ of $\alpha_{\max }$ and $\left\langle j_{\text {ped,max }}\right\rangle$, and the numbers in the figure show the $n$ number of the unstable mode determining the boundary for each model.

deuterium is taken into account self-consistently in both the equilibrium reconstruction and linear stability analysis, but the poloidal rotation, $\Omega_{\theta}$, is included only in the stability analysis for simplicity.

As an example, we show the stability diagram of the JET-ILW \#90287 low- $\nu_{* \mathrm{e}, 95}$ plasma on the $\left(\left\langle j_{\text {ped,max }}\right\rangle, \alpha_{\max }\right)$ plane in figure 1, the diagram which was obtained using the stability criterion $\gamma>0.01 \omega_{\mathrm{A} 0}$; the details, including the plasma profiles, are shown in [17]. Here, $\alpha_{\max }$ is the maximum normalized pressure gradient defined by $\alpha \equiv$ $-\left(\mu_{0} / 2 \pi^{2}\right)\left(\mathrm{d} p_{\mathrm{a} 0} / \mathrm{d} \psi\right)(\mathrm{d} V / \mathrm{d} \psi)\left(V / 2 \pi^{2} R_{0}\right)^{0.5}, \mu_{0}$ is the permeability in the vacuum, $p_{\mathrm{a} 0}$ is the axisymmetric part of the equilibrium plasma pressure $p_{0}, V$ is the volume, $j_{\text {ped,max }}$ is the maximum current density in the pedestal region, $\gamma$ is the growth rate, and $\omega_{A 0}$ is the toroidal Alfvén frequency on axis.
The width of the error bars on the operation point (O.P.) is determined to be $\pm 20 \%$ of $\alpha_{\max }$ and $\left\langle j_{\text {ped, } \max }\right\rangle$, respectively. By analyzing the stability of PBM with different models, it was found that the stability boundary determined with IDEAL is far from the O.P., and the $\omega_{* \mathrm{i}}$ effect moves the boundary away from the O.P., as shown with the DIAwoR result. However, the plasma rotation helps to bring the boundary close to the O.P., and, in fact, the DIAwR boundary is the closest one to the O.P. among the results obtained with the three models.

Figure 2(a) shows the $\nu_{* e, 95}$ dependence of the distance between the stability boundary and O.P., $\Delta_{\alpha}$, which is defined by $\Delta_{\alpha}=\left(\alpha_{\max , \mathrm{BND}}-\alpha_{\max , \mathrm{OP}}\right) / \alpha_{\max , \mathrm{OP}}$. Here $\alpha_{\max , \mathrm{BND}}$ $\left(\alpha_{\max , \mathrm{OP}}\right)$ is the $\alpha_{\max }$ value on the stability boundary (O.P.) where $\left\langle j_{\text {ped,max }}\right\rangle$ is equal to that on the O.P. The error bar of $\Delta_{\alpha}$ is drawn by estimating $\alpha_{\max }$ on the boundary where $\left\langle j_{\text {ped,max }}\right\rangle$ is changed by $\pm 20 \%$ from that on the O.P. The $\Delta_{\alpha}$ value determined with the DIAwoR model is larger than 0.4 in seven shots, but those with the IDEAL and DIAwR models are smaller than 0.25 in almost all of the analyzed shots belonging to the high- $\nu_{* e, 95}$ group. The result implies that both the IDEAL and DIAwR models can be applicable for the ELM stability analysis in the JET-ILW high- $\nu *$ e,95 shots.

However, it should be emphasized that the analyses were performed by identifying the stability with respect to PBM whose $n$ number is up to 100 , hence, it is necessary to confirm whether the $n$ number of the mode determining $\Delta_{\alpha}$ is below 100 or not. The $\nu_{* e, 95}$ dependence of the $n$ number, shown in figure 2(b), indicates that the $n$ number of the mode determining the IDEAL $\Delta_{\alpha}$ is 100 in the shots whose $\nu_{* \mathrm{e}, 95}>0.88$, and the result implies that the IDEAL stability in such shots is restricted by very high- $n$ modes, including the infinite- $n$ ballooning mode. However, such short wavelength modes are usually not regarded as the trigger of the type-I ELM. In fact, the $\omega_{* \mathrm{i}}$ effect stabilizes high- $n$ ballooning modes, and the $n$ number of the mode determining the DIAwoR and DIAwR boundaries is always less than 100. In addition, $\Delta_{\alpha}$ of the shots belonging to the low- $\nu_{* \mathrm{e}, 95}$ group 

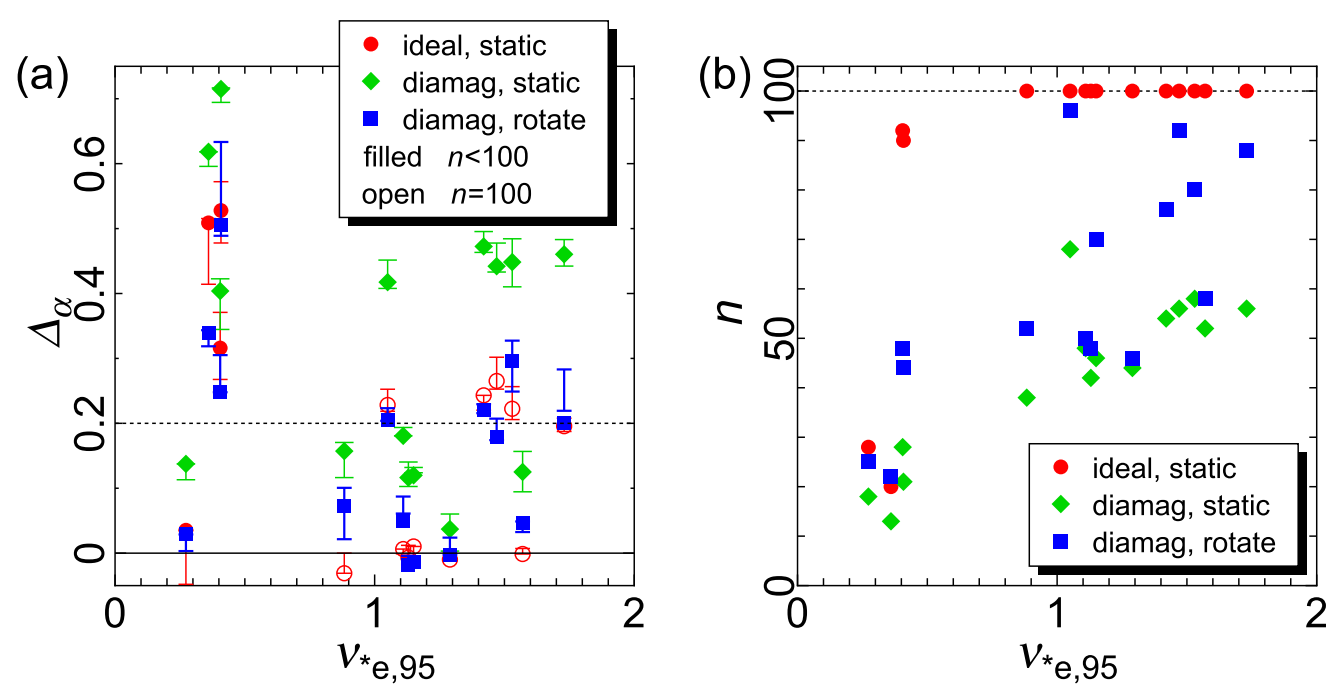

Figure 2. (a) Distance between the operation point and the stability boundary, $\Delta_{\alpha}$, as a function of the collisionality at $\psi=0.95, \nu_{* e, 95}$; the definition of $\Delta_{\alpha}$ is written in the main text. The filled (open) symbol shows the result determined by the $n<100(n=100)$ mode stability. (b) The $n$ number of the most unstable mode determining $\Delta_{\alpha}$ for each model as a function of $\nu_{* e, 95}$.

always become minimum when using the DIAwR model, as shown in figures 1 and 2(a). These results indicate that the $\omega_{* i}$ effect is necessary to analyze the type-I ELM stability from not only a theoretical but also a practical point of view because of a decrease in the $n$ number of the most unstable mode. However, the $\omega_{* i}$ effect stabilizes the ELM too strongly, and hence the destabilizing effect due to rotation is also necessary to determine the stability threshold.

\subsection{Sensitivity of the PBM stability to effective charge in a JET-ILW plasma}

When analyzing the PBM stability numerically, the profile of the bulk ion number density, $N$, is usually determined by

$$
N=\frac{Z-Z_{\mathrm{eff}}}{Z-1} N_{\mathrm{e}}
$$

with the assumption that $Z_{\text {eff }}$ is constant in the plasma, where $Z$ is the charge of impurity. The $Z_{\text {eff }}$ value in JET-ILW is determined from visible bremsstrahlung measurements for a line of sight vertically and horizontally independent, and hence the values are sometimes different. For example, in the JET-ILW \#89709 plasma which belongs to the high- $\nu_{* \mathrm{e}, 95}$ group, $Z_{\text {eff }}$ is estimated as 1.19 when using the horizontal line of sight, which is different from the value estimated with the vertical one as 1.5 . Note that $Z_{\text {eff }}$ estimated with a vertical line is usually more reliable, and hence the stability analyses in the previous subsection were carried out with this value. In this subsection, the sensitivity of the PBM stability to $Z_{\text {eff }}$ is investigated numerically with the JET-ILW \#89709 plasma; the profiles and the ELM stability diagram of the plasma are shown in [17].

When changing $Z_{\text {eff }}$, the number densities of the bulk and impurity ion species become different, as in (15). Such a difference can change not only the $p_{\mathrm{i}}$ but also the bootstrap current and rotation profiles evaluated based on neoclassical theory. Figure 3 shows the profiles of $p,\langle\boldsymbol{j} \cdot \boldsymbol{B}\rangle /\langle B\rangle, \Omega_{\phi}, \Omega_{\theta}$, and $\omega_{* \mathrm{i}}$ evaluated with the different $Z_{\text {eff }}$ values. In the range
$1.2 \leqslant Z_{\text {eff }} \leqslant 1.8$, the profiles of $\langle\boldsymbol{j} \cdot \boldsymbol{B}\rangle /\langle B\rangle$ and $\Omega_{\phi}$ change little, but the changes in the other profiles are visible. It is trivially found that $Z_{\text {eff }}$ affects the pressure gradient, and, in fact, the $\alpha_{\max }$ value on the O.P. changes from 2.28 with $Z_{\text {eff }}=1.2$ to 2.20 and 2.11 with $Z_{\text {eff }}=1.5$ and 1.8 , respectively. It should be emphasized that when increasing $Z_{\text {eff }}, \omega_{* \mathrm{i}}$ becomes smaller due to the lowered $p_{\mathrm{i}}$, but $\Omega_{\theta}$ increases. As discussed in the previous section, plasma rotation can destabilize the PBM, although $\omega_{* \mathrm{i}}$ stabilizes the mode; hence, such a physics trend will make the PBM more unstable.

The stability diagram obtained with different $Z_{\text {eff }}$ values is shown in figure 4(a). Note that the stability is analyzed with the DIAwoR and DIAwR models, because the $n$ number of the mode determining the IDEAL boundary is 100 . When neglecting the rotation effect, the increase of $Z_{\mathrm{eff}}$ brings both the stability boundary and the O.P. to the lower $\alpha_{\max }$ side with a decrease in $\omega_{* \mathrm{i}}$ and $p_{\mathrm{i}}$. The shift of the O.P. is larger than that of the boundary, and as a result, $\Delta_{\alpha}$ tends to become larger with an increase in $Z_{\text {eff }}$, as shown in figure $4(b)$. On the other hand, by including the rotation effect, the boundary moves to the lower $\alpha_{\max }$ side more clearly; namely the rotation can destabilize the PBM effectively as $Z_{\text {eff }}$ increases. In fact, $\Delta_{\alpha}$ decreases from $0.28-0.21$ by increasing $Z_{\text {eff }}$ from $1.2-1.8$, as shown in figure 4(b), which contrasts with the trend observed in the static case. Since $Z_{\text {eff }}$ does not change the $\Omega_{\phi}$ profile, it was found that the sensitivity of the PBM stability to $Z_{\text {eff }}$ appears due to a change in $\omega_{* \mathrm{i}}$ and $\Omega_{\theta}$. In particular, the difference in $\Omega_{\theta}$, which is estimated based on neoclassical theory, destabilizes the ELM enough to change the qualitative dependence of the stability threshold on $Z_{\text {eff }}$.

\section{Nonlinear simulation study of the ELM energy loss in JT-60U rotating plasmas}

In this section, we investigate the nonlinear evolution of the type-I ELM in JT-60U plasmas. As discussed in [23], it was 


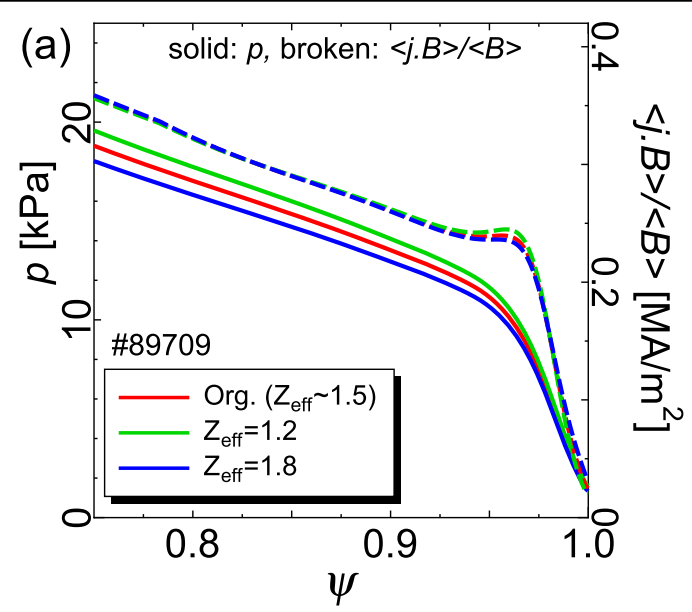

(b)
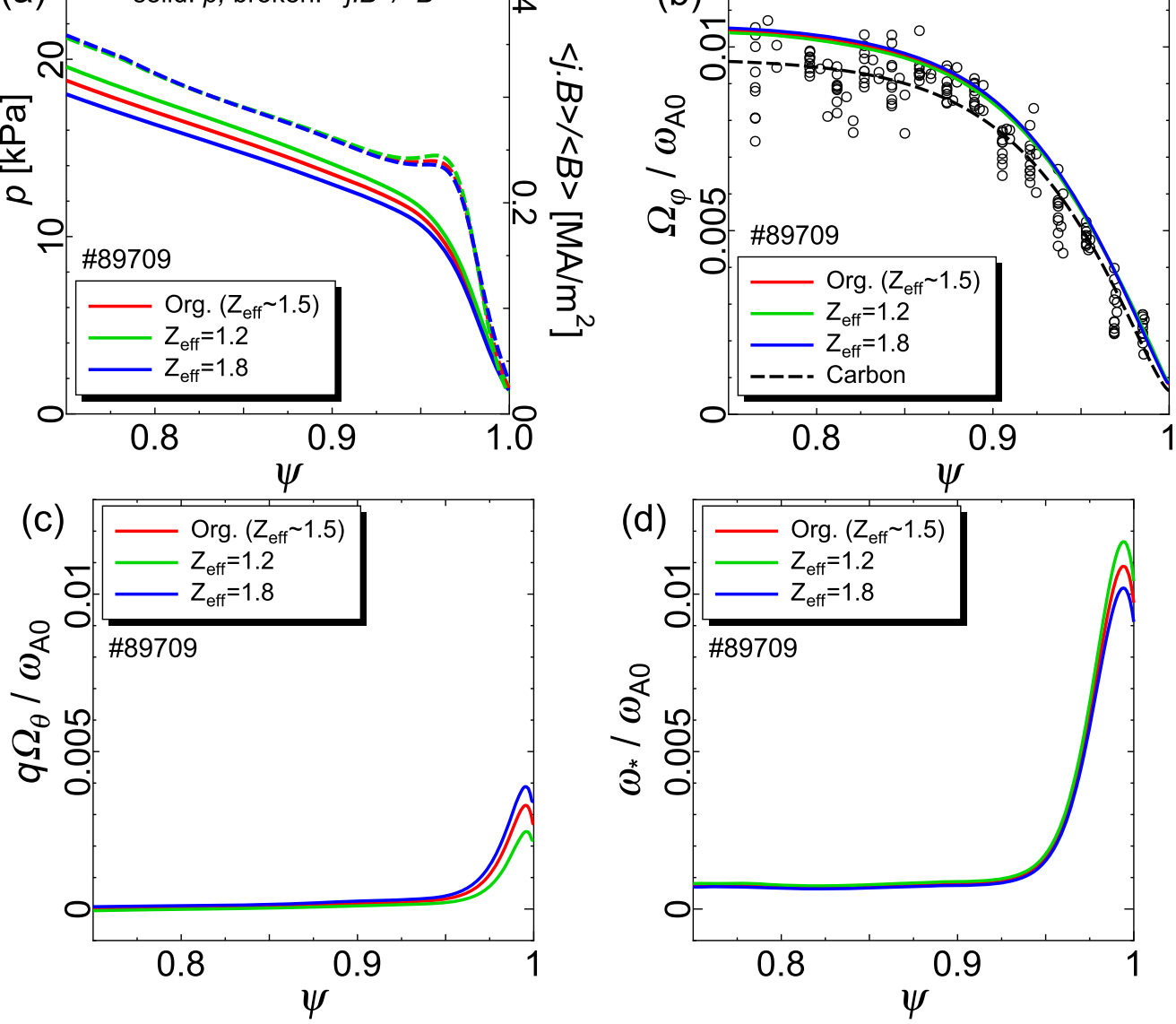

Figure 3. Profiles of the JET-ILW \#89709 high- $\nu_{* e, 95}$ plasma with different $Z_{\text {eff: }}$ (a) $p$ and $\langle\boldsymbol{j} \cdot \boldsymbol{B}\rangle$, (b) $\Omega_{\phi}$, (c) $\Omega_{\theta}$, and (d) $\omega_{* i}$.
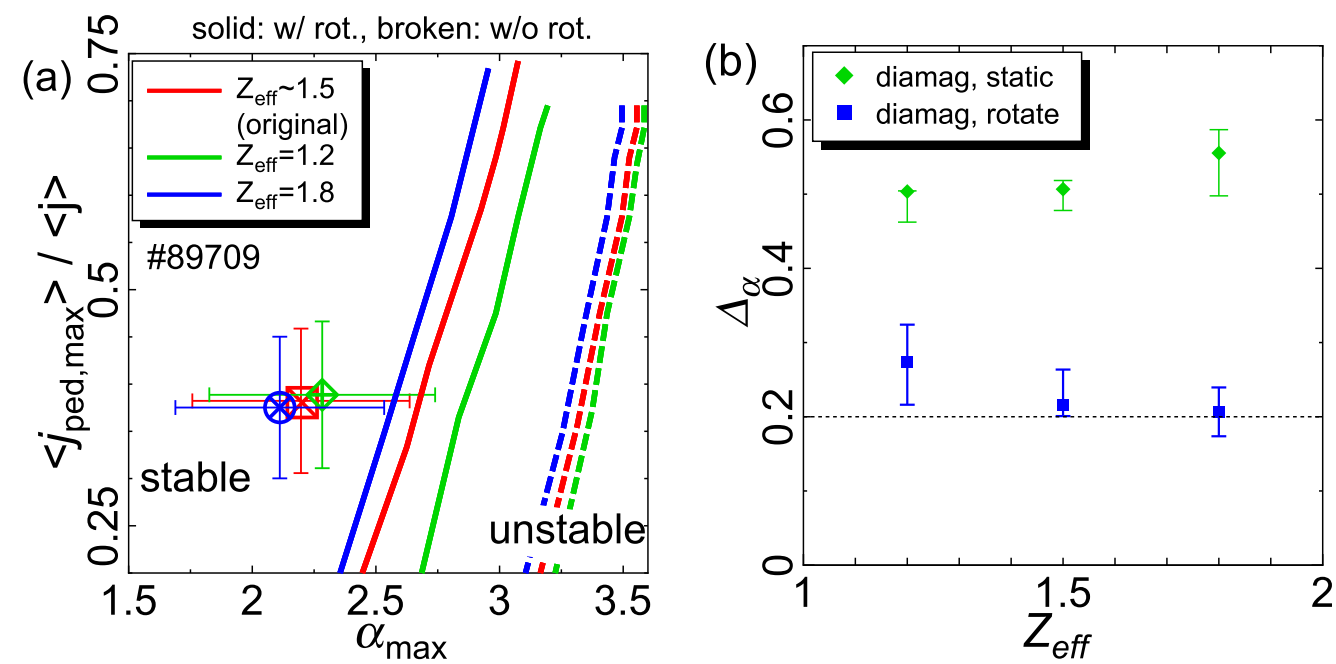

Figure 4. (a) Stability diagram of the JET-ILW \#89709 high- $\nu_{* e}, 95$ plasma on the $\left(\left\langle j_{\text {ped,max }}\right\rangle /\langle j\rangle, \alpha_{\max }\right)$ plane; the stability is analyzed with the DIAwoR and DIAwR models. The O.P.s and stability boundaries are determined with the different $Z_{\text {eff }}$ values. (b) Dependence of $\Delta_{\alpha}$ on $Z_{\text {eff. }}$.

confirmed experimentally that the amount of ELM energy loss, $\Delta W_{\mathrm{ELM}}$, in the plasma rotating in the co-direction of the plasma current is larger than that in the ctr-rotating one in JT-60U. The rotation direction was changed by adjusting the directions of the external momentum input by NBI, and hence other plasma profiles were usually affected simultaneously. In the experiments whose shot numbers are E49228 and E49229, the experimental conditions were set up to minimize such differences in the profiles, except rotation, and, as a result, the ion temperature, $T_{\mathrm{i}}$, profiles were almost the same, which were measured with modulation charge exchange recombination spectroscopy. Unfortunately, the electron 
temperature, $T_{\mathrm{e}}$, profile could not be measured precisely because of the low spatial resolution of Thomson scattering. However, the $T_{\mathrm{e}}$ values near the top of the $T_{\mathrm{i}}$ pedestal were only slightly different, hence the $T_{\mathrm{e}}$ profile could be regarded as similar by assuming $T_{\mathrm{e}}=0.6 T_{\mathrm{i}}$ in both plasmas. The main difference between these plasmas appears in the electron density, $N_{e}$, profiles, which were measured with a lithium beam probe. The $N_{e}$ pedestal in the co-rotating E49228 plasma was clearly located close to the plasma surface; the details of the profiles are presented in [16, 23]. The linear stability of PBM in these plasmas has been discussed in [16], and it was confirmed that the rotation has an impact on the ELM trigger condition in the ctr-rotating plasma, although the condition in the co-rotating one is hardly affected. However, it has still not been identified whether the difference in the $N_{e}$ profiles or the rotation profiles is responsible for the amount of the ELM energy loss. To resolve the problem, the nonlinear ELM evolution and $\Delta W_{\mathrm{ELM}}$ in these plasmas are investigated with the JOREK code. The boundary condition of the simulation is determined with the JT-60U vacuum vessel with the assumption that this acts as an ideal conducting wall, and that of the computational domain in the SOL is the last closed flux surface (LCFS) on which Dirichlet boundary conditions are applied for all variables, except density and temperature, for which Neumann conditions are applied. At the divertor targets, in addition, sheath boundary conditions are used for parallel velocity and energy conduction, and free outflow boundary conditions are applied for the density; the details are described in [18].

The profiles of the plasmas analyzed in this study are the same as those shown in figure 4 in [16], and their experimental conditions are presented in [23]. The ELM characteristic in the co-rotating E49228 plasma is $\Delta W_{\mathrm{ELM}} \simeq 85 \mathrm{~kJ}$ with the frequency of the ELM cycle $f_{\mathrm{ELM}} \simeq 37 \mathrm{~Hz}$, and that in the ctr-rotating E49229 one is $\Delta W_{\mathrm{ELM}} \simeq 45 \mathrm{~kJ}$ with $f_{\mathrm{ELM}} \simeq 45 \mathrm{~Hz}$, respectively. Simulations were run with the full toroidal spectrum from 1-15. As discussed with the linear analyses, the plasmas with the original profiles are stable to PBM even when the rotation effect is included; hence, to make PBM marginally unstable in both plasmas, the pressure at the top of the pedestal was increased by about $10 \%$, although that at the separatrix and the pedestal width were unchanged. Note that the diamagnetic effects were neglected, and only the toroidal rotation was taken into account in the simulations.

Figure 5 shows a comparison of the heat flux to the outer divertor in the co- (E49228) and ctr- (E49229) rotating plasmas. It can be clearly observed that the heat load in the co-rotating plasma is larger than that in the ctr-rotating one, and, in fact, the $\Delta W_{\text {ELM }}$ value evaluated between $0.4 \mathrm{~ms}$ and $0.8 \mathrm{~ms}$ in E49228 is $7.3 \mathrm{~kJ}$, which is about 1.5 times larger than that in E49229 (4.6 kJ between $0.35 \mathrm{~ms}$ and $0.75 \mathrm{~ms}$ ). Unfortunately, in most cases, $\Delta W_{\text {ELM }}$ measured experimentally appears within $1.2 \mathrm{~ms}$ just after the ELM crash, and it is obvious that $\Delta W_{\text {ELM }}$ estimated numerically is much smaller than that in the experiment, even if the simulation period is extended three times longer. However, even though such a quantitative discrepancy remains between the simulation and

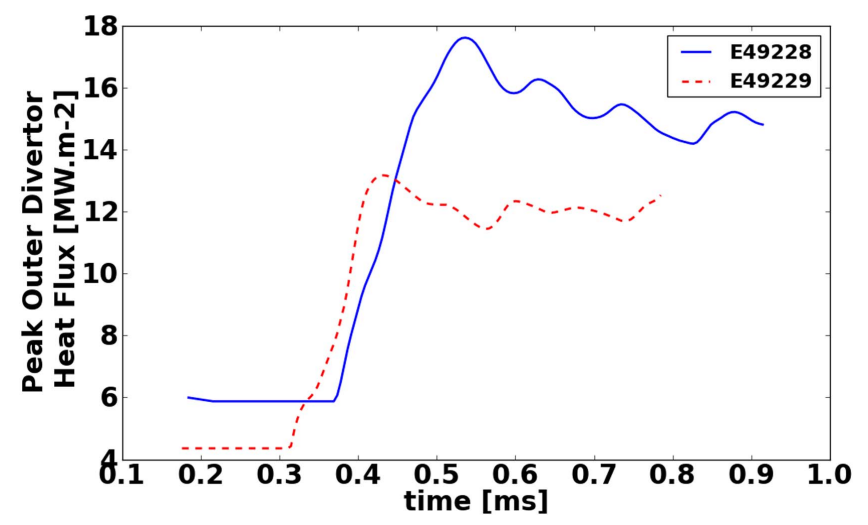

Figure 5. Comparison of peak heat flux to the outer divertor in the JT-60U E49228 (co-rotating) and E49229 (ctr-rotating) plasmas.

experiment, the numerical result successfully captures the qualitative trend observed experimentally, and hence we use it to discuss whether $N_{e}$ or rotation is responsible for changing $\Delta W_{\text {ELM }}$.

To solve the problem, we tried the same simulation by inverting only the rotation direction, and found only small changes in $\triangle W_{\mathrm{ELM}}$. It should be emphasized that the linear stability of PBM does not change when inverting only the toroidal rotation. This result implies that the rotation direction does not play a major role in changing $\Delta W_{\mathrm{ELM}}$ between the E49228 and E49229 plasmas.

Next, we considered the difference in the ELM affected areas observed numerically. Figure 6(a) shows the change of pressure due to the ELM crash, which indicates the ELM affected area. In the colored regions, the amount of lost pressure in the co-rotating plasma is larger than that in the ctrrotating one; namely, the ELM affected area is wider in the co-rotating plasma. One of the candidates which can explain the trend is the difference in the radial width of the linear eigenfunction of the unstable PBM. Figure 6(b) shows a comparison of the eigenfunctions in the co- and ctr-rotating plasmas, which are obtained with the ideal MHD code MINERVA. Note that the $n$ number of the most unstable mode is 12 (15) in the E49228 (E49229) plasma when the $n$ number is truncated from 1-15. As is expected, the radial width of the eigenfunction in the co-rotating plasma is wider, and the colored regions, which show where the eigenfunction has a larger amplitude, are similar to the ELM affected areas in figure 6(a). These results indicate that the linear stability property may play a role in determining $\Delta W_{\text {ELM }}$ emitted in short periods just after the ELM crash, but the difference in the property is not related to the rotation direction. It has been confirmed with the MINERVA code that the linear PBM stability in the E49228 plasma is not affected by the rotation profile, even when it is replaced with the E49229 one. Although the PBM in the E49229 plasma becomes slightly stable when using the E49228 rotation profile, the radial width of the most unstable $n=15$ eigenfunction does not change much. In addition, the $T_{\mathrm{i}}$ and $T_{\mathrm{e}}$ profiles in the E49228 plasma are almost the same as those in the E49229 one. Hence, the plasma density profile, which changes both the pressure and current density profiles, is thought to be 

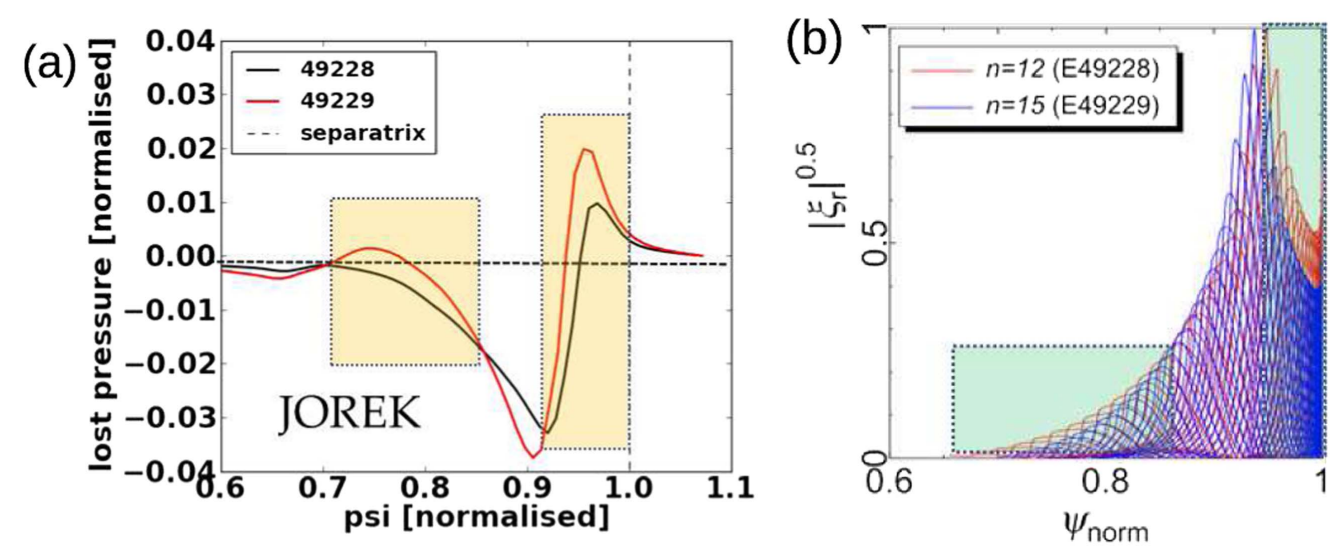

Figure 6. (a) Comparison of the changes of pressure due to the ELM crash, which indicates the ELM affected area in the E49228 (co-rotating) and E49229 (ctr-rotating) plasmas. The colored regions show where the lost pressure in the co-rotating plasma is larger. (b) Comparison of the linear eigenfunctions of the unstable PBM obtained with MINERVA. The colored regions show where the eigenfunction in the E49228 plasma has a larger amplitude; the square root of the amplitude is plotted to emphasize the difference.

responsible for the difference in $\Delta W_{\mathrm{ELM}}$ in the analyzed JT-60U plasmas.

\section{Prediction of pedestal profiles including the rotation and $\omega_{* i}$ effects on ELM stability in JT-60SA}

In this section, the pedestal profiles in JT-60SA are predicted by taking into account the rotation and $\omega_{*_{\mathrm{i}}}$ effects on the PBM stability. Previously, the prediction has been performed based on the EPED1 model [24] with numerical codes from the National Institutes for Quantum and Radiological Science and Technology (QST); the PBM stability has been analyzed under the static plasma assumption with the ideal MHD code MARG2D [25]. In this model, the pedestal width in normalized poloidal flux, $\Delta$, is determined by $\Delta=G\left(\nu_{*}, \epsilon, \ldots\right) \beta_{\theta \text {,ped }}^{0.5}$ by assuming that the pedestal width is constrained by kinetic ballooning mode turbulence, where $G$ is a weakly varying parameter, $\nu_{*}$ is the collisionality, $\epsilon$ is the inverse aspect ratio, and $\beta_{\theta \text {,ped }}$ is the poloidal beta at the top of the pedestal. As discussed in [26], an ensemble average of $G,\langle G\rangle$, obtained with 16 sets of input parameters (four each typical of DIII-D, JET, AUG and ITER), was evaluated as $\langle G\rangle=0.084 \pm 0.010$. In this study, we used the standard value $\langle G\rangle=0.076$, which was determined in the original EPED1 paper [24]. It should be noted that the model with $\langle G\rangle=0.076$ can reproduce well $\Delta$ observed in the DIII-D experiments, but in other tokamaks, the $\langle G\rangle$ value can be different $[27,28]$. In the near edge pedestal region, the plasma current is taken to be dominated by the bootstrap current, which is calculated using the matrix inversion method [29]. The bootstrap current and the pressure profiles are determined by assuming the plasma density and temperature profiles have hyperbolic tangent shapes, as defined in the EPED1 model.

The pedestal height is usually determined by analyzing the linear stability of PBM with a simple model of diamagnetic stabilization as $\gamma_{\mathrm{MHD}}>0.5 \omega_{* p \mathrm{i}}$, where $\gamma_{\mathrm{MHD}}$ is the growth rate of the ideal MHD mode in static plasmas, and $\omega_{* p \mathrm{i}}$ is the half maximum value of the ion diamagnetic frequency in the pedestal. In this study, we have updated the PBM constraint on the pedestal height by using the MINERVA-DI code which can analyze the $\omega_{* \mathrm{i}}$ effect more self-consistently in rotating plasmas.

The rotation profile is predicted with the TOPICS code by solving the momentum balance equation with pinch, diffusion, NBI torque and neoclassical toroidal viscosity (NTV, see e.g. [30]) terms [31-33], where the NTV due to the nonaxisymmetric perturbed magnetic field caused by toroidal field coils is calculated by coupling TOPICS with the threedimensional (3D) equilibrium code VMEC [34] and the 3D non-local neoclassical transport simulation code FORTEC-3D $[35,36]$; the details of the procedure can be found in [32]. Owing to the coupling between TOPICS and D5PM [37], which is the flux-tube five point model of the SOL/divertor plasma, the pressure and temperature profiles can be continuously calculated across the LCFS, and thus these gradients can be estimated. The semi-empirical boundary condition model for toroidal momentum flux is based on the experimental observations in JT-60U, where $E_{r}^{\prime}=0$ at the LCFS gives the toroidal momentum flux at the LCFS [33, 38]. With this value used as the Dirichlet condition for the toroidal momentum solver in TOPICS, a toroidal momentum flux as well as toroidal rotation is solved.

The pedestal profiles are predicted with the EPED model updated by using the MINERVA-DI and TOPICS codes as follows. First, the updated QST's EPED model estimates the profiles of plasma density, temperature and current density under the static assumption, and TOPICS calculates the rotation profiles by using them. After that, the rotation profile is taken into account when analyzing the PBM stability with MINERVA-DI, and the plasma profiles, except rotation, are estimated again. After repeating the iterative process until convergence, all the pedestal profiles are predicted. Note that huge computational resources are required for estimating the NTV, thus, in this study, the rotation profile is estimated without the NTV when predicting the pedestal profiles. However, as discussed in [33], the NTV can help to numerically reproduce the toroidal rotation profile observed 

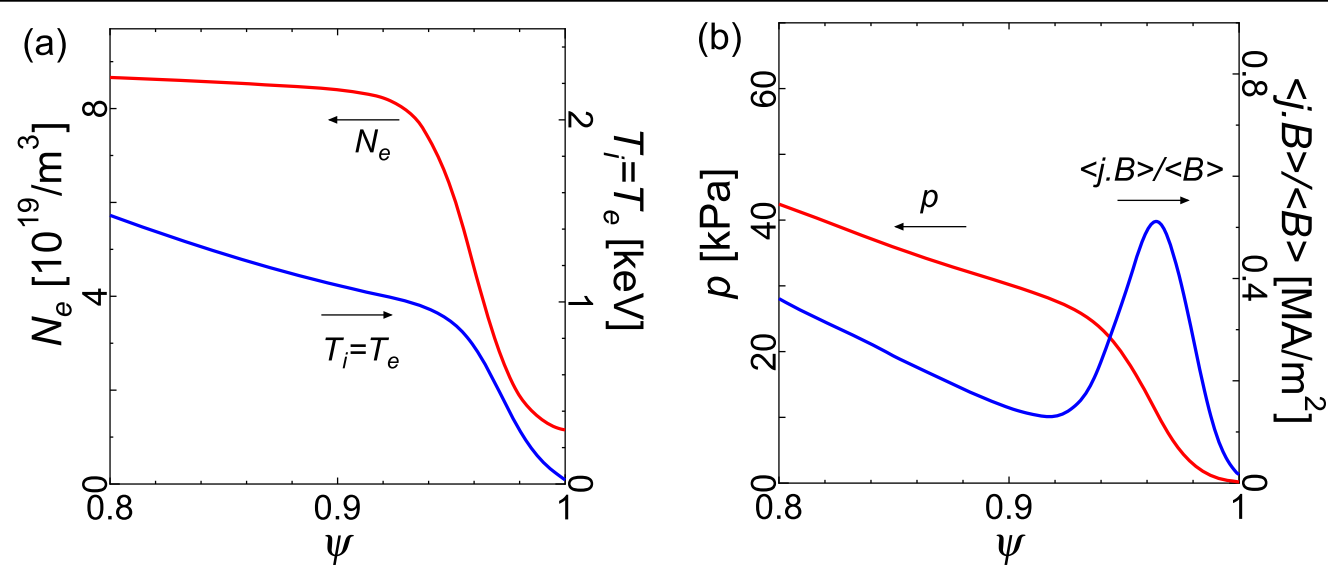

Figure 7. Profiles of the JT-60SA \#4-1 plasma; (a) $N_{\mathrm{e}}$ and $T_{\mathrm{i}}\left(=T_{\mathrm{e}}\right)$, (b) $p$ and $\langle\boldsymbol{j} \cdot \boldsymbol{B}\rangle$.
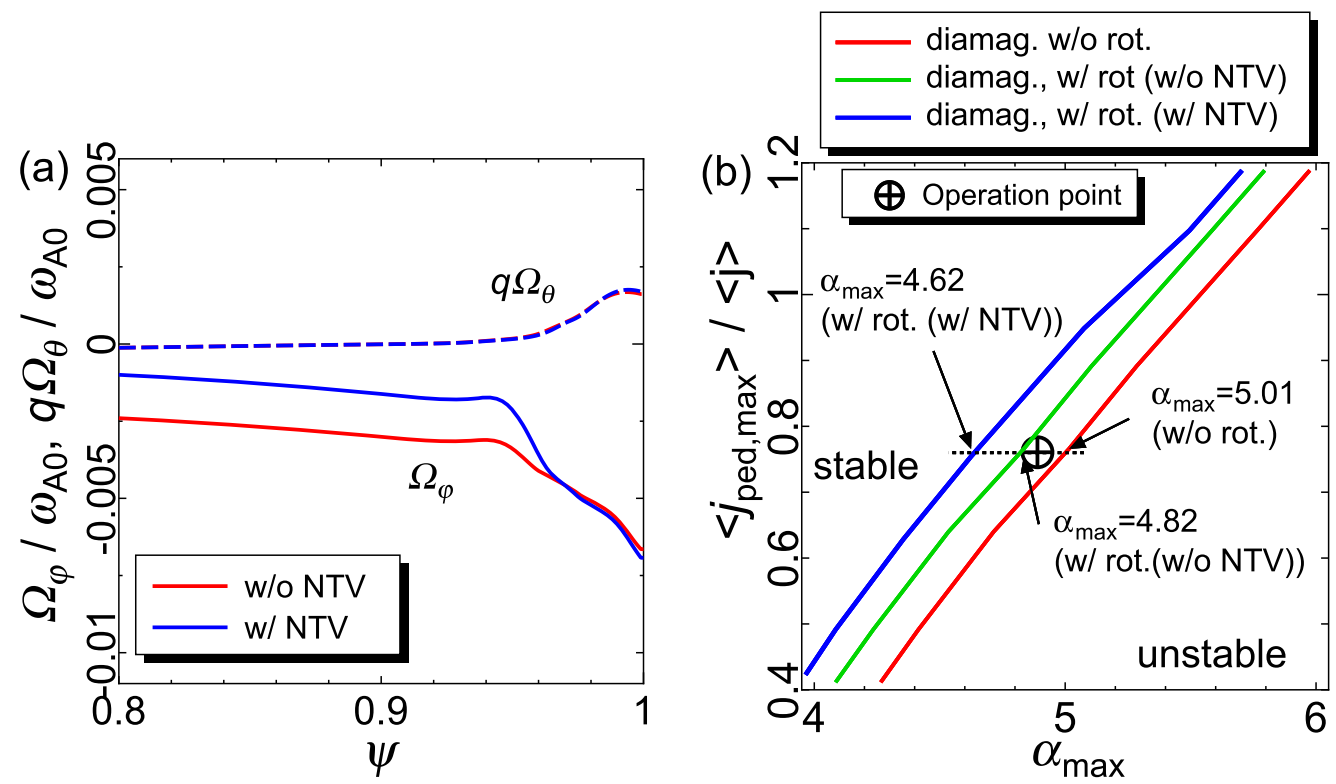

Figure 8. (a) Profiles of $\Omega_{\phi}$ and $\Omega_{\theta}$ calculated with and without the NTV. (b) Stability diagram of the JT-60SA \#4-1 plasma on the $\left(\left\langle j_{\text {ped,max }}\right\rangle /\langle j\rangle, \alpha_{\max }\right)$ plane. The stability boundaries are determined with and without the rotation effect; the $\omega_{* \mathrm{i}}$ effect is always included. The boundaries determined, including rotation, are obtained by using the rotation profiles estimated with and without the NTV, respectively.

experimentally. Therefore, the PBM stability is analyzed again by replacing the rotation profile calculated, including the NTV, to confirm whether or not the change in the rotation profile affects the ELM trigger condition.

The target plasma analyzed in this study belongs to the JT-60SA plasma operation scenario \#4-1, which is called an ITER-like shape inductive scenario [39-41]. The plasma parameters are $B_{t 0}=2.28 \mathrm{~T}, I_{\mathrm{p}}=4.6 \mathrm{MA}, R_{0}=2.94 \mathrm{~m}$, $a=1.14 \mathrm{~m}, \kappa_{95} \simeq 1.7, \delta_{95} \simeq 0.4$, and $\beta_{\mathrm{N}} \simeq 2.8$, respectively. Since the scenario has a target parameter $N_{e} / N_{\mathrm{GW}}>0.8$, the height of the density pedestal is determined first as $N_{\text {e,ped }}=8.19 \times 10^{19} \mathrm{~m}^{-3}$, where $N_{\mathrm{GW}}$ is the Greenwald density; in this plasma, $N_{\mathrm{GW}}=1.12 \times 10^{20} \mathrm{~m}^{-3}$. Hence, the height of the pressure pedestal is adjusted by changing that of the $T_{\mathrm{i}}$ pedestal with the assumption that $T_{\mathrm{i}}=T_{\mathrm{e}}$. Note that the boundary condition for the stability analysis is assumed as an ideal conducting wall located at the the positions of the vacuum vessel and stabilizing plate [39].
Figure 7 shows the profiles of $N_{e}$ and $T_{\mathrm{i}}\left(=T_{\mathrm{e}}\right)$, and $p$ and $\langle\boldsymbol{j} \cdot \boldsymbol{B}\rangle$ predicted with the updated QST's EPED model, respectively; in this case, the height of the temperature pedestals is $1.03 \mathrm{keV}$. The plasma with these profiles is stable to the ideal MHD modes whose $n$ number is less than 20, even when assuming a no-wall condition, and hence the wall stabilizing effect can be regarded as negligible, although the JT-60SA high- $\beta_{\mathrm{N}}$ scenario plasmas should be stabilized with the stabilizing plate.

Based on this reference equilibrium, the impact of plasma rotation on the ELM trigger condition is investigated. The rotation profiles estimated with the profiles of this equilibrium are shown in figure 8(a) for the cases with and without the NTV. As shown in this figure, the NTV changes the toroidal rotation profile inside from the inflection point of the pressure pedestal, but does not affect the poloidal one. By drawing the stability diagram on the $\left(j_{\text {ped,max }}, \alpha_{\text {max }}\right)$ plane, it was confirmed that the O.P. is located on the stability boundary 
determined, including the rotation predicted without the NTV, as shown in figure $8(\mathrm{~b})$. The stability boundary slightly moves to the higher $\alpha_{\max }$ side when neglecting rotation, but the difference in $\alpha_{\max }$ between the stability boundaries near the O.P. is only about $5 \%$; note that the $\omega_{* \mathrm{i}}$ effect is always taken into account in the stability analysis. When the rotation is estimated including the NTV, on the other hand, the PBM becomes more unstable, and the boundary moves to the lower $\alpha_{\max }$ side. As a result, the difference in $\alpha_{\max }$ between the boundaries determined with and without rotation, including the NTV, is about $10 \%$ near the O.P. Such a difference directly affects the plasma performance, because, as is wellknown, the edge pedestal height mainly determines the boundary conditions for the core plasma. In fact, the height of the temperature pedestals should be decreased to be below $1 \mathrm{keV}$ to stabilize the PBM. Hence, it is necessary to predict the pedestal profiles by using the latest knowledge and numerical codes, and, as one of the examples, this study shows that the stability analysis, including the rotation and $\omega_{* i}$ effects, contributes to a more precise prediction. It should be emphasized that the JT-60SA plasma predicted in this study still has a large $\alpha_{\max }$ value, even when the rotation effect is taken into account. We think this is a promising result for achieving the target parameters of the JT-60SA scenario \#4-1.

\section{Summary and discussion}

The stability with respect to PBM in JET, JT-60U, and future JT-60SA plasmas was investigated numerically with two extended MHD simulation codes, MINERVA-DI and JOREK. MINERVA-DI can identify the linear stability of MHD modes with the $\omega_{* i}$ effect in rotating tokamak plasmas by solving the extended Frieman-Rotenberg equation corresponding to the diamagnetic MHD model. JOREK allows us to simulate nonlinear ELM dynamics with rotation, $\omega_{*_{\mathrm{i}}}$, resistivity, viscosity, and diffusivities by solving the five-field reduced MHD equations.

First, the linear stability of PBM in JET-ILW was analyzed, including plasma rotation and the $\omega_{*_{\mathrm{i}}}$ effects with MINERVA-DI. By comparing the PBM stability threshold determined numerically with the ELM trigger condition observed experimentally in 14 JET-ILW shots, it was found that the ELM trigger condition can be explained when both the rotation and $\omega_{* \mathrm{i}}$ effects are taken into account in the numerical stability analysis. This is because the $\omega_{* \mathrm{i}}$ effect is required to reasonably determine the wavelength of the PBM, and the rotation effect is necessary for destabilizing the PBM by reducing and/or overcoming the stabilization due to $\omega_{* \mathrm{i}}$. The sensitivity of the PBM stability to the effective charge, $Z_{\text {eff }}$, was also investigated with a JET-ILW plasma, and the results identified that the stability can be affected by $Z_{\text {eff }}$ mainly due to a change in the poloidal rotation which is evaluated based on neoclassical theory.

Next, the amount of ELM energy loss, $\Delta W_{\mathrm{ELM}}$, in the JT-60U plasmas rotating in opposite directions was simulated with finite resistivity and viscosity by using JOREK. In the experiments, it was reported that $\Delta W_{\mathrm{ELM}}$ in the plasma rotating in the co-direction of the plasma current is larger than that in the ctr-rotating one, and the JOREK simulation qualitatively reproduced the experimental physics trend. By comparing the ELM-affected areas determined by the nonlinear simulation with the radial profiles of the eigenfunction obtained by the ideal linear analysis, it was confirmed that the difference in the linear stability property, due not to the rotation direction but to the plasma density profile, is responsible for changing $\Delta W_{\mathrm{ELM}}$ emitted in the short period just after the ELM crash. From the viewpoint of the linear MHD stability, the radial width of the PBM eigenfunction becomes narrower when the current density near the plasma surface decreases, because the current-driven component of the PBM stability becomes smaller. At the edge pedestal region, the current density profile is mainly composed of the bootstrap current which is roughly proportional to the pressure gradient profile, and, as shown in figure 4(c) in [16], the current density near the plasma surface in the ctr-rotating plasma is smaller than that in the co-rotating one due to the difference in the plasma density profile. We think this is the main reason why the plasma density profile is responsible for changing the linear stability property.

After establishing quantitative understandings regarding the PBM stability property, including the rotation and $\omega_{* \mathrm{i}}$ effects, we predicted the pedestal profiles in the future JT-60SA plasma. The procedure for the prediction basically follows the EPED1 model, but the MHD stability, determining the pedestal height, is analyzed with MINERVA-DI to take into account the above effects. In this study, the pedestal of the JT-60SA plasma operation scenario \#4-1, the ITER-like shape inductive scenario, was predicted with a rotation profile estimated with the TOPICS code. It was shown that the plasma rotation estimated, including the neoclassical toroidal viscosity, destabilizes the PBM and degrades the pedestal performance by about $10 \%$ compared to that determined without rotation, but the pressure pedestal height will be high enough to achieve the target parameters required for the scenario.

There is much work remaining in the future. For example, although the type-I ELM trigger condition can be explained by the linear stability analysis with extended MHD models, as discussed in this study and several recent papers, it is necessary to find the key physics causing the large amount of ELM energy loss comparable to the experimental value in JT-60U. One of the candidates is the ELM onset determined by the nonlinear MHD stability with multi harmonics, whose quantitative importance was recently reported in JET simulations with JOREK [18]. Such simulations using JT-60U plasmas are ongoing, and the results will be reported in the near future. Further quantitative studies regarding the rotation and $\omega_{* \mathrm{i}}$ effects on the ELM stability in JET, JT-60U and other present experiments are necessary to complete the validation of their importance, and the results will help to more precisely predict the ELM trigger condition in future experimental devices and reactors, including JT-60SA, ITER and DEMO. At present, electromagnetic torque has not been taken into account in rotation predictions with the collaborative framework among TOPICS, VMEC and FORTEC-3D, though it has been reported in $[42,43]$ that electromagnetic torque 
plays a role, especially on the rational surfaces. Estimating the torque requires a numerical code under development, and the predictions of rotation profiles with the torque in JT-60SA will be revisited. A predictive study of plasma profiles in the whole region in JT-60SA is also necessary to verify whether the plasma parameters satisfy the targets required for each scenario or not. An integrated simulation with CRONOS is ongoing to predict the profiles of the scenario \#4-1 plasma by using the pedestal profiles predicted in this study, and the result will be presented in the near future.

\section{Acknowledgments}

The authors would like to thank S Ide and G Giruzzi for providing an opportunity for the collaborative study between the EU and Japan. The authors are grateful to Drs Y Kamada, M Yagi, N Oyama, C F Maggi, E de la Luna and J Garcia for their beneficial comments and suggestions. Some of the authors (NA and $\mathrm{MH}$ ) gratefully acknowledge contributions from Drs S Satake and Y Suzuki in the NTV calculations. This work was partially supported by JSPS KAKENHI Grant Number 15K06656, and has been partly carried out within the framework of the EUROfusion Consortium and has received funding from the Euratom research and training programme 2014-2018 under grant agreement No 633053. The views and opinions expressed herein do not necessarily reflect those of the European Commission. The computations were partly carried out using the HELIOS supercomputer system at IFERC-CSC, Aomori, Japan under the Broader Approach collaboration between Euratom and Japan, implemented by Fusion for Energy and Japan. The MARCONI supercomputer at CINECA in Italy was also used to analyze JET plasmas.

\section{ORCID iDs}

N Aiba (iD https://orcid.org/0000-0002-0652-2892

\section{References}

[1] Snyder P B, Wilson H R, Ferron J R, Lao L L, Leonard A W, Osborne T H, Turnbull A D, Mossessian D, Murakami M and Xu X Q 2002 Phys. Plasmas 92037

[2] Saarelma S et al Contributors to the EFDA-JET Work Programme 2005 Plasma Phys. Control. Fusion 47713

[3] Aiba N, Furukawa M, Hirota M, Oyama N, Kojima A, Tokuda S and Yagi M 2011 Nucl. Fusion 51073012

[4] Maggi C F et al JET contributors 2015 Nucl. Fusion 55113031

[5] Snyder P B et al 2007 Nucl. Fusion 47961

[6] Ferraro N M, Jardin S C and Snyder P B 2010 Phys. Plasmas 17102508

[7] Xu X Q, Dudson B D, Snyder P B, Umansky M V, Wilson H R and Casper T 2011 Nucl. Fusion 51103040

[8] Aiba N, Tokuda S, Furukawa M, Snyder P B and Chu M S 2009 Comput. Phys. Commun. 1801282
[9] Aiba N 2016 Plasma Phys. Control. Fusion 58045020

[10] Huysmans G T A and Czarny O 2007 Nucl. Fusion 47659

[11] Dudson B D, Umansky M V, Xu X Q, Snyder P B and Wilson H R 2009 Comput. Phys. Commun. 1801467

[12] Sovinec C R, Glasser A H, Gianakon T A, Barnes D C, Nebel R A, Kruger S E, Schnack D D, Plimpton S J, Tarditi A, Chu M S and the NIMROD Team 2004 J. Comput. Phys. 195355

[13] Brennan D P, Kruger S E, Schnack D D, Sovinec C R and Pankin A 2006 J. Phys.: Conf. Ser. 4663

[14] Park W, Belova E V, Fu G Y, Tang X Z, Strauss H R and Sugiyama L E 1999 Phys. Plasmas 61796

[15] Park G et al The CPES Team 2007 J. Phys.: Conf. Ser. 78 012087

[16] Aiba N, Honda M and Kamiya K 2017 Nucl. Fusion 57 022011

[17] Aiba N et al 2017 Nucl. Fusion 57126001

[18] Pamela S J P et al 2017 Nucl. Fusion 57076006

[19] Shirai H, Barabaschi P, Kamada Y and the JT-60SA Team 2017 Nucl. Fusion 57102002

[20] Hazeltine R D and Meiss J D 1992 Plasma Confinement (Redwood City, CA: Addison-Wesley)

[21] Sauter O, Angioni C and Lin-Liu Y R 1999 Phys. Plasmas 62834

[22] Sauter O, Angioni C and Lin-Liu Y R 2002 Phys. Plasmas 9 5140 (erratum)

[23] Kojima A, Oyama N, Sakamoto Y, Kamada Y, Urano H, Kamiya K, Fujita T, Kubo H, Aiba N and the JT-60 Team 2009 Nucl. Fusion 49115008

[24] Snyder P B et al 2009 Nucl. Fusion 49085035

[25] Aiba N, Tokuda S, Ishizawa T and Okamoto M 2006 Comput. Phys. Commun. 175269

[26] Snyder P B, Groebner R J, Hughes J W, Osborne T H, Beurskens M, Leonard A W, Wilson H R and Xu X Q 2011 Nucl. Fusion 51103016

[27] Leyland M J et al (JET-EFDA contributors) 2015 Nucl. Fusion 55013019

[28] Dunne M G et al (The EUROfusion MST1 Team) 2017 Plasma Phys. Control. Fusion 59025010

[29] Kikuchi M and Azumi M 1995 Plasma Phys. Control. Fusion 371215

[30] Shaing K C 2003 Phys. Plasmas 101443

[31] Honda M, Ide S, Takizuka T, Hayashi N, Yoshida M, Yagi M and Fujita T 2013 Nucl. Fusion 53073050

[32] Honda M, Satake S, Suzuki Y, Matsunaga G, Shinohara K, Yoshida M, Matsuyama A, Ide S and Urano H 2014 Nucl. Fusion 54114005

[33] Honda M et al 2015 Nucl. Fusion 55073033

[34] Hirshman S P and van RIJ W I 1986 Comput. Phys. Commun. 43143

[35] Satake S, Kanno R and Sugama H 2008 Plasma Fusion Res. 3 S1062

[36] Satake S, Sugama H, Kanno R and Park J 2011 Plasma Phys. Control. Fusion 53054018

[37] Hayashi N, Takizuka T and Hosokawa M 2007 J. Nucl. Mater. 363-365 1044

[38] Kamiya K, Honda M, Urano H, Yoshida M, Kamada Y, Itoh K and JT-60 team 2014 Phys. Plasmas 21122517

[39] Kamada Y, Barabaschi P, Ishida T S and JT-60SA Research Plan contributors 2013 Nucl. Fusion 53104010

[40] JT-60SA Research Unit 2016 JT-60SA Research Plan (http:// www.jt60sa.org/pdfs/JT-60SA_Res_Plan.pdf) v3.3

[41] Giruzzi G et al 2017 Nucl. Fusion 57085001

[42] Pustovitov V D 2011 Nucl. Fusion 51013006

[43] Liu Y, Connor J W, Cowley S C, Ham C J, Hastie R J and Hender T C 2012 Phys. Plasmas 19102507 